\title{
Thermocapillary migration mechanism of molten silicon droplets on horizontal solid surfaces
}

\author{
Tao SUN ${ }^{1}$, Cunhua JIANG ${ }^{1}$, Jianning DING ${ }^{1,2, *}$, Ningyi YUAN ${ }^{1,2, *}$ \\ ${ }^{1}$ School of Mechanical Engineering, Changzhou University, Changzhou 213164, China \\ ${ }^{2}$ Collaborative Innovation Center of Photovoltaic Science and Engineering, the Breeding Construction Point of State Key Laboratory of \\ Photovoltaic Engineering Science, Changzhou 213164, China
}

Received: 02 March 2017 / Revised: 15 April 2017 / Accepted: 23 April 2017

(C) The author(s) 2017. This article is published with open access at Springerlink.com

\begin{abstract}
Effective lubrication under extreme conditions such as high temperature is of considerable importance to ensure the reliability of a mechanical system. New lubricants that can endure high temperatures should be studied and employed as alternatives to traditional oil-based lubricant. In this paper, a thermocapillary model of a silicone-oil droplet is developed by solving the Navier-Stokes and energy equations to obtain the flow, pressure, and temperature fields. This is accomplished using a conservative microfluidic two-phase flow level set method designed to track the interface between two immiscible fluids. The numerical simulation accuracy is examined by comparing the numerical results with experimental results obtained for a silicone-oil droplet. Hence, the movement and deformation of molten silicon droplets on graphite and corundum are numerically simulated. The results show that a temperature gradient causes a tension gradient on the droplet surface, which in turn creates a thermocapillary vortex. As the vortex develops, the droplet migrates to the low-temperature zone. In the initial stage, the molten silicon droplet on the corundum substrate forms two opposite vortex cells, whereas two pairs of opposite vortices are formed in the silicone fluid on the graphite substrate. Multiple vortex cells gradually develop into a single vortex cell, and the migration velocity tends to be stable. The greater the basal temperature gradient, the stronger the internal thermocapillary convection of the molten silicon droplet has, which yields higher speeds.
\end{abstract}

Keywords: molten silicon; droplet; temperature gradient; thermocapillary migration

\section{Introduction}

Effective lubrication is a key factor in ensuring the stability of a high-temperature machine such as a gas turbine or silicon pulling machine [1,2]. In such machine systems, temperatures $T$ higher than $1,000{ }^{\circ} \mathrm{C}$ can develop between the components. In these scenarios, traditional oil-based lubricant is not appropriate for use, because of its disadvantages such as unfavorable viscosity-temperature $\mu-T$ characteristics and flammability [3,4]. Thus, new lubricants suitable for such applications should be found and studied. For example, meltable materials are promising lubricants that can function under the above conditions. Recently, Li et al. [5] exploited the high thermal diffusivity of liquid metal to utilize this material as a lubricant under extreme pressure $p$.

Thermocapillarity refers to phenomena that arise because of variation in the interfacial tension at a fluid-solid interface, which is caused by a $T$ gradient. Thermocapillarity occurring between a friction pair will affect the lubrication performance by changing the surface tension $\sigma$ and meniscus shape. Previously, Brozska et al. [6] described the thermocapillary migration behavior of droplets on solid surfaces using a lubrication model, proving that the migration velocity was linearly

* Corresponding authors: Jianning DING, E-mail: dingjn@cczu.edu.cn; Ningyi YUAN, E-mail: nyyuan@cczu.edu.cn 


$\begin{array}{ll}\text { List of symbols } \\ H, W & \text { Rectangular width and height } \\ \theta & \text { Static contact angle } \\ T_{\mathrm{H}} & \text { The temperature of high temperature side } \\ x & \text { Abscissa displacement } \\ T_{\mathrm{c}} & \text { The temperature of low temperature } \\ \mathrm{Bo} & \text { Bond } \\ \mathrm{Ca} & \text { Capillary } \\ \mathrm{Re} & \text { Reynolds } \\ \mathrm{Oh} & \text { Ohnesorge numbers } \\ \rho & \text { Density } \\ G & \text { Gravitational acceleration } \\ V & \text { Droplet velocity } \\ L & \text { Characteristic length } \\ D & \text { The droplet contact diameter under the } \\ & \quad \text { solid surface. } \\ k^{-1} & \text { Capillary length } \\ \mathrm{Ml}, \mathrm{ua} & \text { The dynamic viscosity of droplet and air } \\ C_{\mathrm{pl},} C_{\mathrm{pa}} & \text { Specific heat of droplet and air } \\ k_{\mathrm{l}}, k_{\mathrm{a}} & \text { Thermal conductivity of droplet and air } \\ F_{x}, F_{y} & \text { Surface tension forces in the } x \text { and } y \\ & \quad \text { directions }\end{array}$

$\gamma_{\mathrm{T}} \quad$ The surface tension coefficient

$\sigma_{0} \quad$ Surface tension at the reference temperature $T_{0}$

$\Omega_{1} \quad$ Air subdomain

$\Omega_{2} \quad$ Droplet subdomain

$\phi \quad$ Level set function

$\varepsilon \quad$ Interface thickness

$\gamma \quad$ Reinitialization parameter

u Velocity vector

$b_{1 \mathrm{~s}} \quad$ Slip length

$u_{\tau} \quad$ Velocity of the fluid slip on the boundary

Ma Marangoni number

$\Delta T \quad$ Temperature difference between two contact lines

$\alpha \quad$ The thermal diffusivity

$\Delta p \quad$ The droplet pressure difference

$R \quad$ Droplet interface curvature

$\sigma_{\mathrm{LG}}, \sigma_{\mathrm{SG}}, \sigma_{\mathrm{SL}}$ Gas-liquid surface tension, solid-air interfacial tension, solid-liquid interfacial tension dependent on the radius $r$ and that a certain critical $r$ existed. In addition, Brochard [7] assumed an approximately wedge-shaped droplet with a small static contact angle $\theta$, and found that the droplet motion varies with $\theta$. Tseng et al. [8] analyzed the change in the flow and $T$ fields inside the droplet; however, good consistency between experimental and numerical results was not obtained because of differences in the initial and experimental conditions. Finally, Chen et al. [9] studied the effect of contact-angle hysteresis and slip length on the migration velocity during droplet migration, showing that the magnitude of the slip coefficient has little effect on the migration velocity.

A lubrication system will generate heat and, therefore, thermocapillary motion; then, the $\mu, p$, and capillary motion of the lubricant will change during the droplet migration. Therefore, the thermocapillary migration of molten-material-based lubricant has a significant influence on the lubrication efficacy [10, 11]. However, few works report the flow characteristics of molten materials under high T. Among the investigated materials, silicon has a high melting point and is easily oxidized at high $T$ values. However, it is difficult to study the thermocapillary migration phenomena of molten silicon droplets via an experimental approach.

In this work, we employ the finite element method with the two-phase level set technique, which is ideally suited to the tracking of interfaces between two immiscible fluids, to examine thermocapillary migration in molten silicon droplets. To verify the accuracy of the conserved level set method, the numerical simulation results obtained for a silicone-oil droplet are compared with experimental results. Hence, the movement and deformation of molten silicon droplets on graphite and corundum substrates are numerically simulated.

Graphite crucibles and corundum crucibles are two commonly used base materials for molten silicon. In this study, high- $T$ contact-angle equipment is used to measure the $\theta$ of the silicon liquid on the graphite substrate and corundum, in an argon atmosphere. The evolution of the internal $T$ field and the flow field of the molten silicon droplets is studied based 
on the experimental value of $\theta$. The thermocapillary migration behavior of the silicon droplets on the graphite and corundum substrates under the argon atmosphere is investigated via numerical simulation, which provides a theoretical basis for control of the thermocapillary convection.

\section{Physical model and meshing}

In the numerical simulation conducted in this study, a small liquid droplet was placed at the bottom solid wall in a rectangular cross-sectional area with dimensions of $1.5 \mathrm{~mm} \times 8 \mathrm{~mm}$ (height $H \times$ width $W$ ). The liquid droplet was initially assumed to have a cylindrical cap shape, with a static $\theta$ which is defined as the angle formed between the droplet/air interface and the droplet/solid interface at the intersection of droplet-air-solid phase (Fig. 1(a)). A uniform temperature gradient $G$ was imposed on the bottom wall, and the $T$ distribution followed the relation $T=T_{\mathrm{H}}-$ $G \times x$. Where $T_{H}$ is the temperature of high temperature side, $x$ is the abscissa displacement. Here, if $x=0, T=$ $T_{\mathrm{H}}$, and if $x=W, T=T_{\mathrm{C}}$. Here, $T_{\mathrm{C}}$ is the temperature of low temperature side. As the droplet was considered to be very small, the density of the liquid within it could be assumed to be constant and the influence of the body force could be neglected. Note that both the droplet and air can be regarded as incompressible

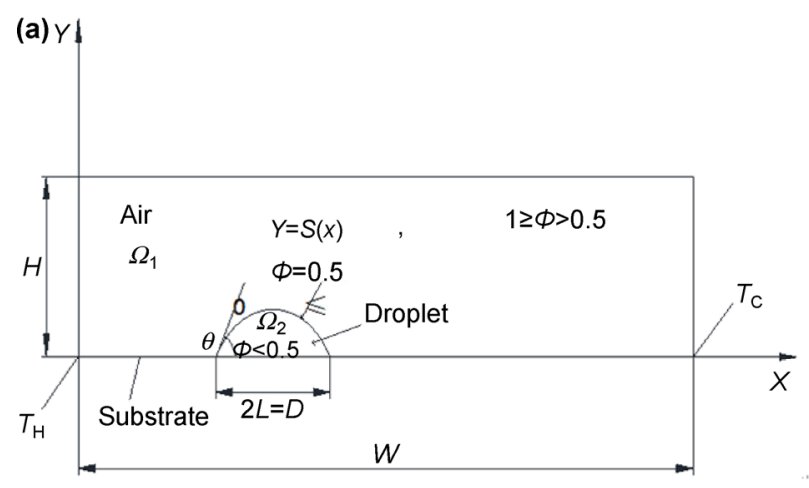

(1)

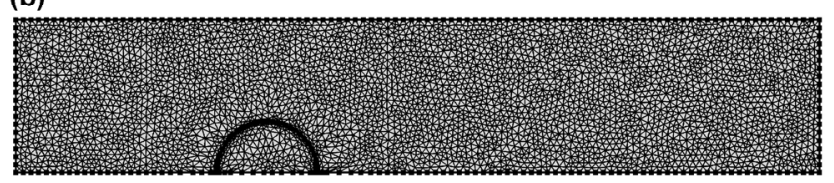

Fig. 1 (a) Theoretical model of thermocapillary migration. (b) Schematic meshing of model. and Newtonian fluids. Further, $\sigma$ and $\theta$ are functions of $T$, whereas the physical properties of the droplet and air are independent of $T$.

A total of 18,912 grids were used for calculation in the subsequent simulation, and grid encryption was used at the interface, as shown in Fig. 1(b). The bond, capillary, Reynolds, and Ohnesorge numbers, which are expressed as follows, were used to determine the cylindrical cap assumption:

$$
\begin{gathered}
\mathrm{Bo}=\frac{\rho g r^{2}}{\sigma} ; \mathrm{Ca}=\frac{\mu \cdot v}{\sigma} ; \mathrm{Re}=\frac{\rho v L}{\mu} ; \mathrm{We}=\frac{\rho v^{2} L}{\sigma} ; \\
\mathrm{Oh}=\frac{\mu}{\sqrt{\rho \sigma L}}=\frac{\sqrt{\mathrm{We}}}{\operatorname{Re}}
\end{gathered}
$$

For silicone-oil droplets with $r=0.5 \mathrm{~mm}$, the migration velocity has an order of magnitude of $1 \mathrm{~mm} / \mathrm{s}$. We obtained $\mathrm{Bo}=0.115, \mathrm{Ca}=0.01, \mathrm{Re}=10, \mathrm{We}=10^{-5}$, $\mathrm{Oh}=10^{-4}$ where $\rho$ represents the droplet density; $g$ is the gravitational acceleration; $v$ represents the droplet velocity; $L$ is the characteristic length; and $D$ represents the droplet contact diameter under the solid surface.

If the droplet $D$ is smaller than the capillary length $k^{-1}, k^{-1}=\sqrt{(\sigma / \rho g)^{1 / 2}}$ and the shape of the side surface of the droplet ridge has a circular cross section. However, if $D>k^{-1}$, the shape of the liquid drop ridge is flat.

In the silicone-oil case examined in this study, thermocapillary migration was simulated under the condition that $\theta=90^{\circ}$. For the molten silicon on graphite and corundum substrates, the thermocapillary migration of the droplets was simulated under the conditions that $\theta=45^{\circ}$ (hydrophilic condition) and $\theta=90^{\circ}$.

\section{Mathematical formulation and boundary conditions}

\subsection{Gas-liquid two-phase flow of thermocapillary mathematical model}

The two-dimensional equations for the conservation of mass, momentum, and energy for incompressible and Newtonian fluids are expressed as

$$
\rho_{i}\left[\frac{\partial u}{\partial x}+\frac{\partial v}{\partial y}\right]_{i}=0
$$




$$
\begin{gathered}
\rho_{i}\left[\left(\frac{\partial u}{\partial t}+u \frac{\partial u}{\partial x}+v \frac{\partial u}{\partial y}\right)\right]_{i}=-\frac{\partial p}{\partial x}+\mu_{i}\left[\left(\frac{\partial^{2} u}{\partial x^{2}}+\frac{\partial^{2} u}{\partial y^{2}}\right)\right]_{i}+F_{x} \\
\rho_{i}\left[\left(\frac{\partial v}{\partial t}+u \frac{\partial v}{\partial x}+v \frac{\partial v}{\partial y}\right)\right]_{i}=-\frac{\partial p}{\partial y}+\mu_{i}\left[\left(\frac{\partial^{2} v}{\partial x^{2}}+\frac{\partial^{2} v}{\partial y^{2}}\right)\right]_{i}+F_{y} \\
\rho_{i} C_{p_{i}}\left[\left(\frac{\partial T}{\partial t}+u \frac{\partial T}{\partial x}+v \frac{\partial T}{\partial y}\right)\right]_{i}=k_{i}\left[\left(\frac{\partial^{2} T}{\partial x^{2}}+\frac{\partial^{2} T}{\partial y^{2}}\right)\right]_{i}
\end{gathered}
$$

where $u_{i}$ and $v_{i}$ are the velocity components in the $x$ and $z$ directions, respectively; $\rho_{i}$ is the fluid density; $\mu_{i}$ is the dynamic viscosity; $C_{\mathrm{p} i}$ is the specific heat; and $k_{i}$ is the thermal conductivity. The subscripts $i=l$, $a$ are used to represent liquid and air, respectively. $F_{x}$ and $F_{y}$ are the surface tension forces in the $x$ and $y$ directions, respectively.

The force generated by the $\sigma$ differences at the gas-liquid interface is given by the equation

$$
\mu \frac{\partial u}{\partial y}=\gamma_{\mathrm{T}} \frac{\partial T}{\partial x}
$$

Here, $\mu$ is the dynamic viscosity and $\gamma_{\mathrm{T}}$ is the surfacetension coefficient, which can be assumed to vary linearly with temperature [12] such that

$$
\sigma=\sigma_{0}-\gamma_{\mathrm{T}}\left(T-T_{0}\right)
$$

where $\sigma_{0}$ is the surface tension at the reference temperature $T_{0}$. The physical properties of the liquid and gas are listed in Table 1.

\subsection{Gas-liquid interface tracking method}

The conservative level set $[13,14]$ method is commonly used to manage the deformation of the free interface

Table 1 Physical properties of fluids (silicone-oil, air at $25^{\circ} \mathrm{C}$, molten silicone, $\operatorname{Ar}$ at $1,427^{\circ} \mathrm{C}$ ).

\begin{tabular}{ccccc}
\hline $\begin{array}{c}\text { Physical } \\
\text { property }\end{array}$ & Silicone-oil & Air & $\begin{array}{c}\text { Molten } \\
\text { silicon }\end{array}$ & Ar \\
\hline$\rho\left(\mathrm{kg} / \mathrm{m}^{3}\right)$ & 968 & 1.1614 & 2560 & 0.28 \\
$\sigma(\mathrm{N} / \mathrm{m})$ & 0.021 & - & 0.72 & - \\
$\gamma_{\mathrm{T}}(\mathrm{N} /(\mathrm{m} \cdot \mathrm{K}))$ & $6 \times 10^{-5}$ & - & $8 \times 10^{-5}$ & - \\
$\mu(\mathrm{Pa} \cdot \mathrm{s})$ & 0.02 & $1.74 \times 10^{-5}$ & $7.5 \times 10^{-4}$ & $7.35 \times 10^{-5}$ \\
$k(\mathrm{~W} /(\mathrm{m} \cdot \mathrm{K}))$ & 0.1 & $2.63 \times 10^{-2}$ & 0.21 & $5.7 \times 10^{-2}$ \\
$C_{\mathrm{p}}(\mathrm{J} /(\mathrm{kg} \cdot \mathrm{K}))$ & 1670 & 1007 & 2800 & 521 \\
\hline & & & & \\
\hline
\end{tabular}

during droplet motion. In this method, the air $\Omega_{1}$ and droplet $\Omega_{2}$ subdomains are separated by the interface $\varphi$ with the level set function $\varphi=0.5$. The value of $\varphi$ smoothly increases from 0 to 1 with $0 \leq \varphi<0.5$ in the liquid-droplet subdomain, with $0.5<\varphi \leq 1$ in the air subdomain. The reinitialized convection of the interface can be expressed as

$$
\frac{\partial \varphi}{\partial t}+\boldsymbol{u} \cdot \nabla \varphi=\gamma \nabla \cdot\left(\varepsilon \nabla \varphi-\varphi(1-\varphi) \frac{\nabla \varphi}{|\nabla \varphi|}\right.
$$

where $\varepsilon$ is the interface thickness, $\gamma$ is the reinitialization parameter, and $u$ is the velocity vector. Note that $\gamma$ determines the numerical calculation of the convergence in this case and should be of the same order of magnitude as the maximum flow rate in the flow field.

\subsection{Boundary conditions}

The left and right walls are nonslip adiabatic walls. The upper wall is isothermal with no sliding property; the bottom wall is a wetting surface with a uniform $G$. The Navier slip condition is applied at the liquid-solid boundary, such that

$$
u_{\tau}=b_{1 \mathrm{~s}} \frac{\partial u}{\partial y}
$$

where $b_{\mathrm{ls}}$ is the slip length and $u_{\tau}$ is the velocity of the fluid slip on the boundary.

\section{Results and discussion}

The droplet velocity $v$ is defined as the velocity of the droplet motion relative to the solid surface. The method of numerical simulation used to calculate $v$ gives the average velocity, which is obtained by integration of the velocities of each point inside the droplet, followed by division by the volume integral of the entire droplet.

To verify the accuracy of the conservative level set method, the numerical simulation results obtained for a silicone-oil droplet were compared with the experimental results. On the basis of this numerical calculation method, the thermocapillary migration processes of molten silicon droplets on graphite and corundum substrates were studied. The thermocapillary 
migration process for droplets primarily includes a dynamic change in the gas-liquid two-phase flow field, a $T$ change of the droplets and the surrounding gas, and deformation of the gas-liquid interface.

\subsection{Analysis and validation of silicone-oil droplet migration model}

\subsubsection{Slip length effect}

Figure 2 illustrates the change in the silicone-oil migration rate over time $t$ for slip lengths $b=1,3$, and $5 \mathrm{~nm}$. In the initial stage $(t<0.5 \mathrm{~s})$, the greater the value of $b$, the greater the droplet migration rate has. When the migration velocity becomes stable, the effect of $b$ on the silicone-oil migration rate decreases. The stable migration velocity of the silicone oil tends to be consistent for all three examined $b$ values. Previous research has shown that the $b$ of this model has little effect on the thermocapillary migration rate [8], and the computational model used to obtain the results presented below was unified for all cases using $b=1$.

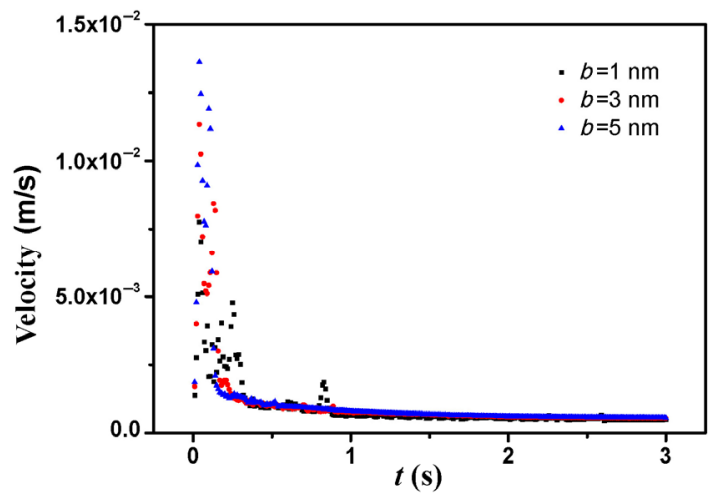

Fig. 2 Effect of slip length on silicone-oil migration velocity.

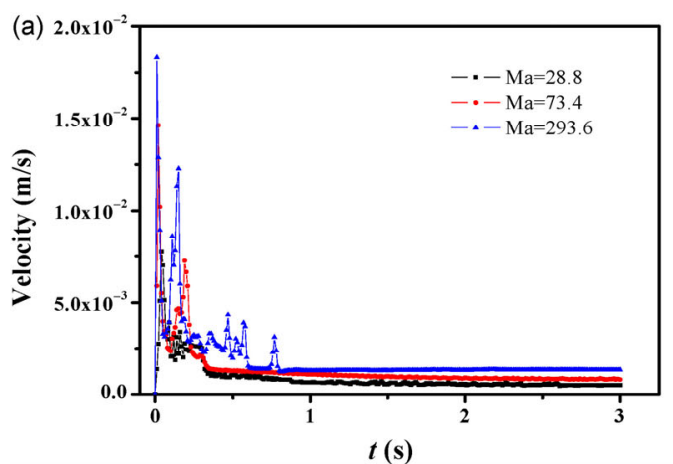

\subsubsection{Temperature gradient effect}

Figure 3(a) illustrates the $G$ effect on the silicone-oil migration, as determined for $G=1.96,5$, and $20 \mathrm{~K} / \mathrm{mm}$ (the corresponding Marangoni (Ma) numbers are 28.8, 73.4, and 293.6, respectively), $\theta=90^{\circ}$, and the droplet contact diameter $D=1 \mathrm{~mm}$. It is apparent that the average migration rate changes with $t$. The Ma effect (i.e., the thermocapillary convection intensity) is usually expressed by the Ma numbers [15], where

$$
\mathrm{Ma}=\gamma_{\mathrm{T}} \Delta T L / \mu \alpha
$$

Here, $\Delta T$ is the temperature difference between two contact lines, $L$ is the contact length between the droplet and bottom wall, and $\alpha$ is the thermal diffusivity. A larger Ma number indicates greater internal thermocapillary convection intensity. As shown in Fig. 3(a), the silicone-oil droplet thermocapillary migration velocity first increases rapidly, before gradually decreasing to a steady process. The greater the value of $G$, the greater the maximum $v$ and the shorter the time taken to reach the maximum $v$; this behavior indicates that the instantaneous acceleration of the droplet migration increases as $G$ increases. This is because the variation in $\sigma$ from lower values on the warm side of the interface to higher values on the cool side generates a flow in the droplet, which exerts a hydrodynamic force on the solid surface that points in the direction of the applied G. The solid surface, which is held immobile, exerts an equal and opposite reaction on the droplet that propels it in the opposite direction [16], i.e., toward cooler regions. Under suitable conditions, quasisteady motion, for

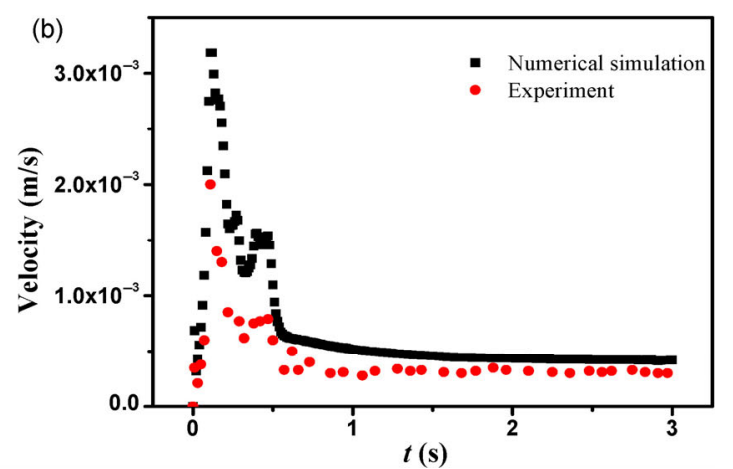

Fig. 3 (a) Silicone-oil migration velocity under different temperature gradients $G$ with Marangoni Ma numbers of 28.8, 73.4, and 146.8. (b) Comparison of computational fluid dynamics (CFD) and experimental results for silicone-oil migration velocity at $G=1.96 \mathrm{~K} / \mathrm{mm}$ and contact angle $\theta=40^{\circ}$. 
which the net hydrodynamic force on the droplet is zero, can be achieved.

Equation (6) shows that the shear stress produced by the thermocapillary effect on the gas-liquid interfacial surface is proportional to $G$. These trends are consistent with experimental phenomena [17].

In the numerical simulation, the boundary conditions were chosen such that the static $\theta=40^{\circ}$ and the substrate $G$ was $2 \mathrm{~K} / \mathrm{mm}$; these conditions are identical to those reported for a previous experiment conducted by Tseng [8]. Figure 3(b) illustrates the numerical and experimental results for the thermocapillary migration of the silicone-oil droplet. A certain degree of consistency is apparent between the different sets of results, which verifies the accuracy of the conservation level set method. Therefore, the numerical simulation can provide basic and detailed physical quantities for the droplets, such as the flow, $p$, and $T$ fields during the migration. However, because the numerical simulation does not consider the effect of buoyancy convection and gravity, certain inconsistencies exist between the numerical and experimental results.

\subsubsection{Detailed flow and temperature fields inside silicone- oil droplet}

Figure 4 illustrates the variation in the internal $T$ distribution of the silicone-oil droplet over time, for $\mathrm{Ma}=146.8, b_{\mathrm{ls}}=1 \mathrm{~nm}, \theta=90^{\circ}, G=20 \mathrm{~K} / \mathrm{mm}$, and $D=$ $1 \mathrm{~mm}$. The colored lines indicate isotherms, and the $T$ difference between two adjacent isotherms is $0.8 \mathrm{~K}$. The initial stage of the droplets and the $T$ of the surrounding gas constitute the reference temperature. With increasing $t$, heat is transferred from the substrate to the droplets and air. Because the thermal diffusion coefficient of silicone oil is greater than that of air, heat is transmitted faster in the droplets and eventually forms the obtained $T$ distribution. As shown in Fig. 5 $(t=0.01 \mathrm{~s})$, in the high- $T$ region (left side), the inner vortex is greater; this is attributed to the greater $G$. Two vortices are formed in the gas area around the droplets, and development of this asymmetric vortex pushes the droplet toward the low- $T$ region.

In contrast to the internal flow field of the droplet shown in Fig. 6, the $T$ and $V$ fields are almost decoupled at $0.01 \mathrm{~s}$, and the $T$ field does not reflect the disturbance
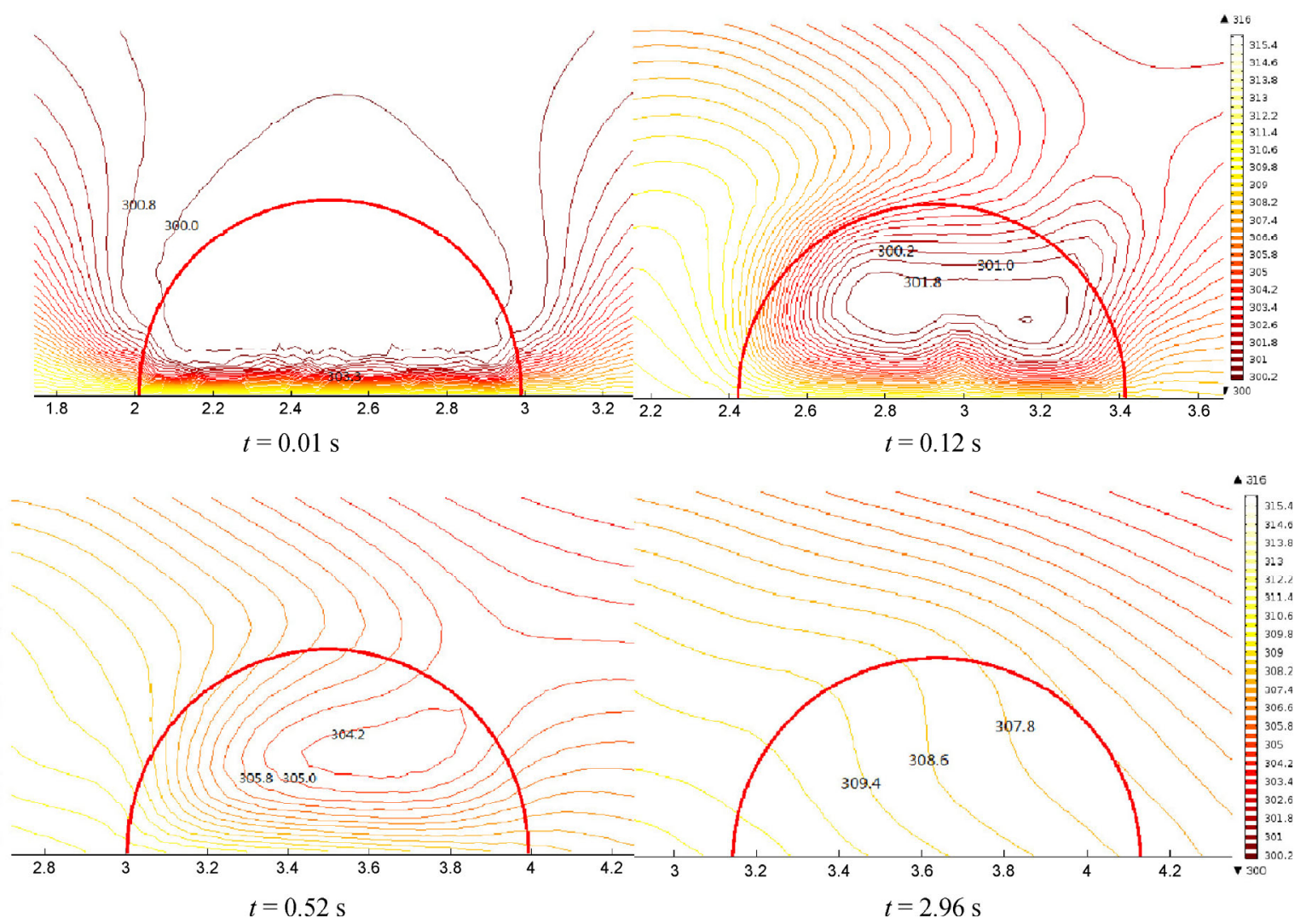

Fig. 4 Temperature $T$ changes in silicone oil with time $t$ at $\theta=90^{\circ}$ and $G=20 \mathrm{~K} / \mathrm{mm}$. 


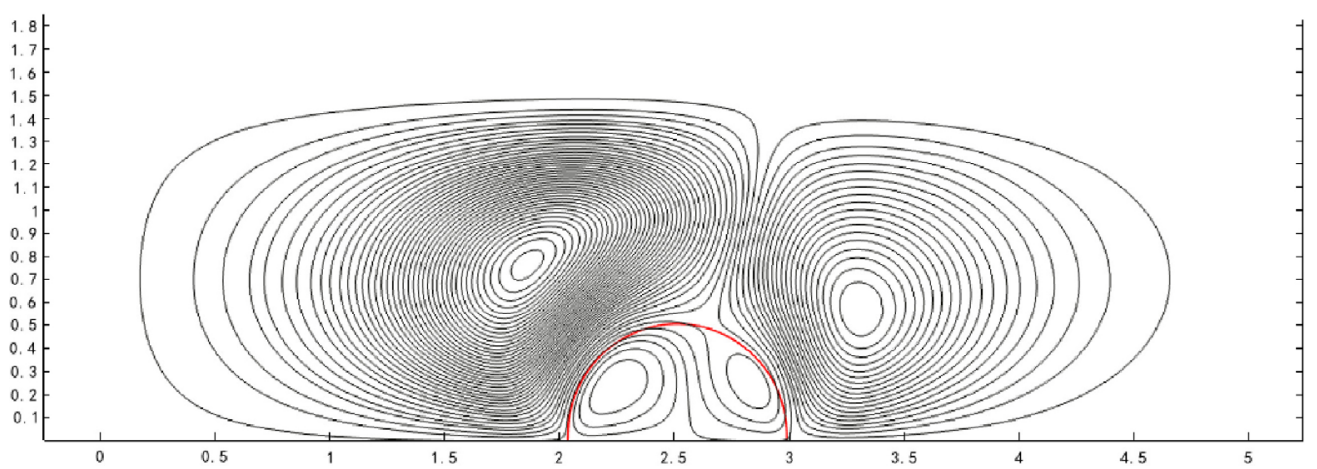

Fig. 5 Silicone-oil droplet streamlines for $\theta=90^{\circ}$ and $G=20 \mathrm{~K} / \mathrm{mm}$ at $t=0.01 \mathrm{~s}$.
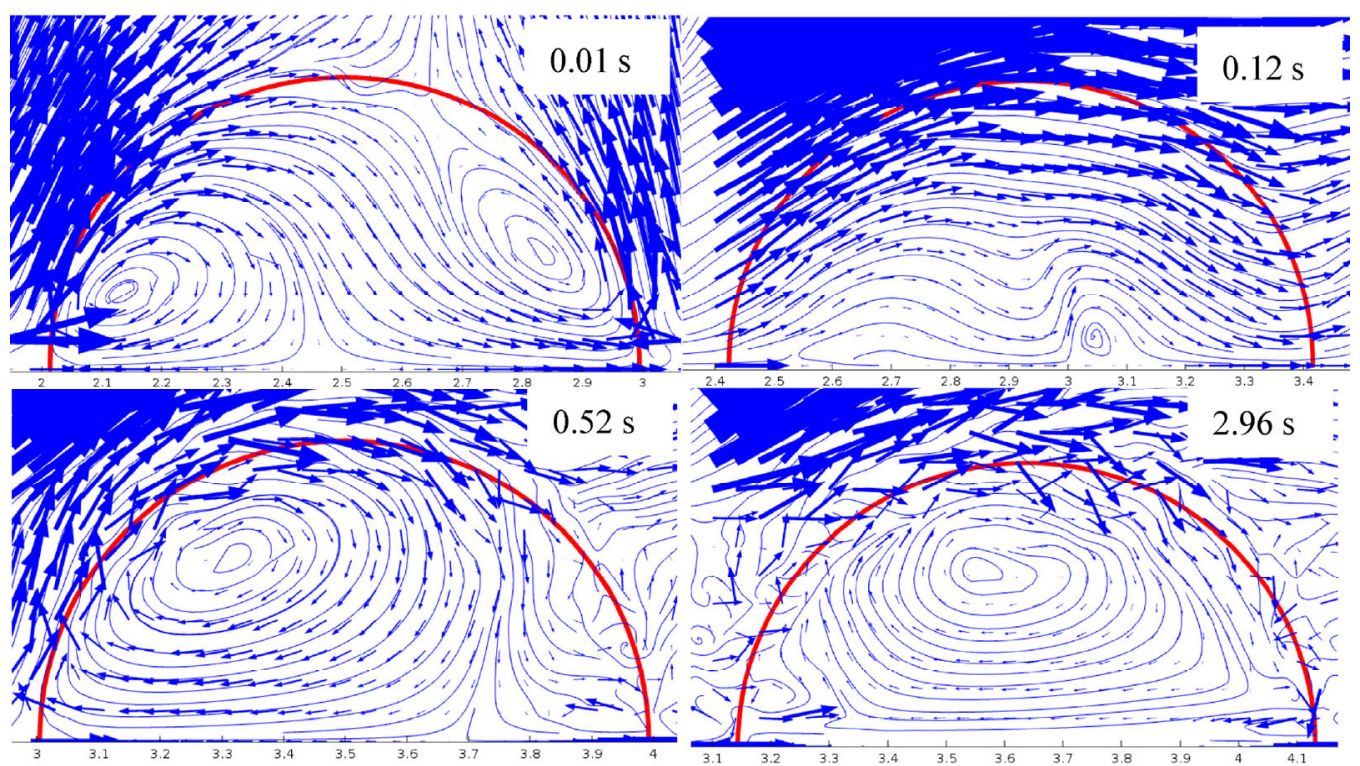

Fig. 6 Flow field inside silicone oil at $\theta=90^{\circ}$ and $G=20 \mathrm{~K} / \mathrm{mm}$.

caused by convection. With increasing $t$, the $T$ no longer decreases linearly, and Fig. 4 shows that the convective torsion caused by the flow is distorted. Figure 6 demonstrates that with increasing $t$, the development of the inner turbines on the high- $T$ side increases. On the low- $T$ side, however, the final two vortices develop into a vortex and the silicone-oil droplet migration velocity is stable.

Figure 7 illustrates the droplet internal and gasliquid interface $T$ distributions at different times. In the initial stage, the highest $T$ is at the lowest part of the droplet. Then, as the front and rear contact points move, the position of the advancing angle contact point becomes the low- $T$ point of the free interface. At a certain $t$, the lowest point of the interface $T$ is obtained at the forward angle contact point and remains constant.

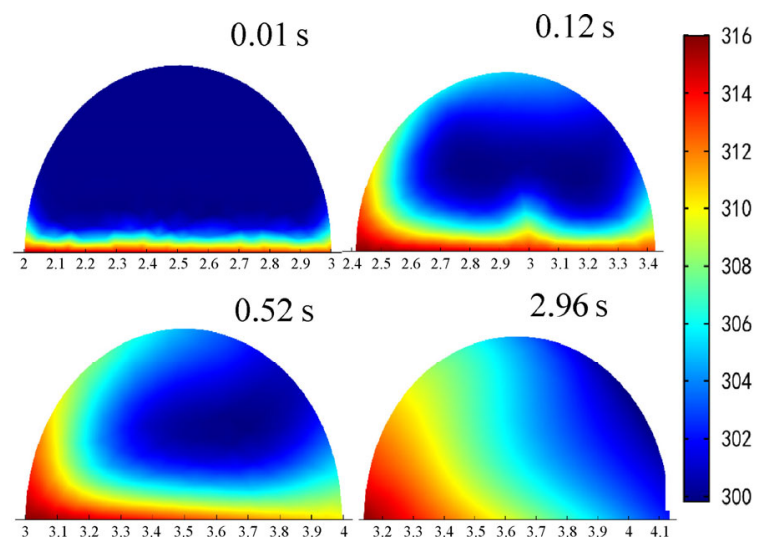

Fig. 7 Temperature distribution in silicone-oil process at $\theta=$ $90^{\circ}$ and $G=20 \mathrm{~K} / \mathrm{mm}$.

\subsubsection{Deformation and variation of dynamic contact angle during migration process}

Figure 8 shows the deformation process for droplets 

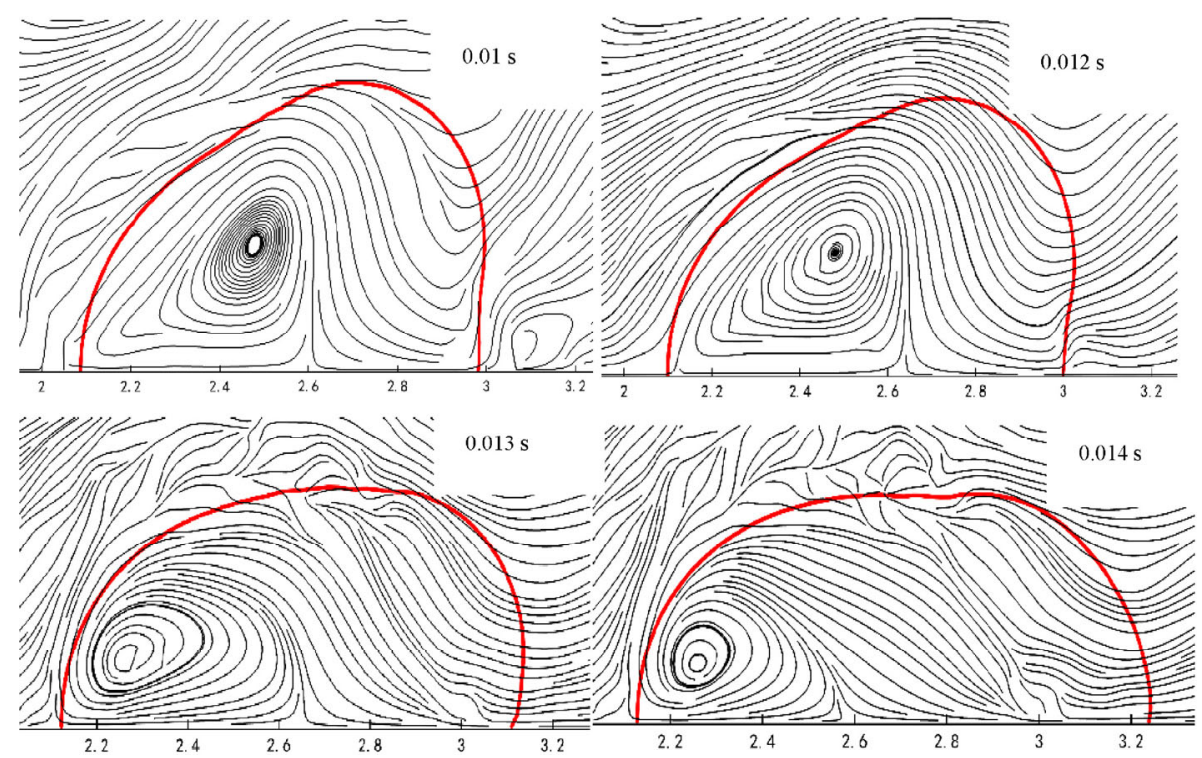

Fig. 8 Change of interface shape during silicone oil migration.

under boundary conditions of $\theta=90^{\circ}$ and $G=20 \mathrm{~K} / \mathrm{mm}$. The non-uniform distribution of the gas-liquid interface generates differences in the gas-liquid surface $p$ that deform the droplet.

From the current simulation, $\theta$ increases during the migration process and then stabilizes at a fixed value, while the advancing angle is always greater than the receding angle. It can be seen from Fig. 9 that the advancing and receding angles [18] increase before $t=$ $0.01 \mathrm{~s}$, because the droplet $\sigma$ gradient produces shear stress that deforms the interface. Moreover, changes in the interface curvature vary the internal and external differential $p$ values, thereby altering $\theta$. The gas-liquid interfacial deformation yields capillary convection within the droplet. Note that the capillary flow is caused by the different curvatures at the droplet interface. According to the Young-Laplace equation,

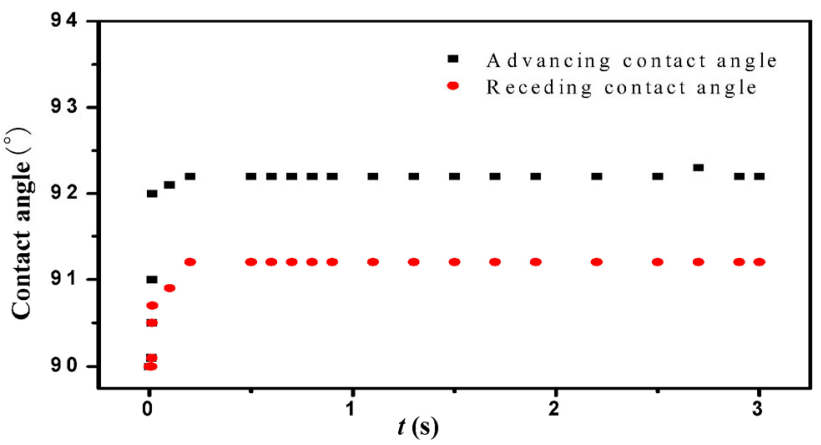

Fig. 9 Receding/advancing dynamic contact angles of silicone oil droplet. for droplets,

$$
\Delta p=\frac{2 \sigma}{R}
$$

where $\Delta p$ represents the droplet pressure difference and $R$ is the droplet interface curvature. The droplet deformation during the migration induces the difference in the interface curvature at the droplet interface. The smaller the curvature, the greater the difference is between the inside and outside $p$ values. Therefore, the capillary flow occurs from the smaller side of the curvature to the larger side, and the droplet interface shape affects the capillary convection direction. Figure 8 demonstrates that the capillary convection direction is opposite and resistant to the thermocapillary convection (Marangoni convection); this behavior ultimately stabilizes the advancing and receding angles at a constant value.

\subsection{Numerical simulation of thermocapillary migration of molten silicon droplets}

Figure 10 shows the changes in the static $\theta$ values of the silicon liquid on the graphite and corundum surfaces in response to changing $T$. Here, $\theta$ decreases rapidly as $T$ increases from the silicon melting point to $1,700 \mathrm{~K}$.

The Young equation [19] is given by:

$$
\sigma_{\mathrm{LG}} \times \cos \theta=\sigma_{\mathrm{SG}}-\sigma_{\mathrm{SL}}
$$




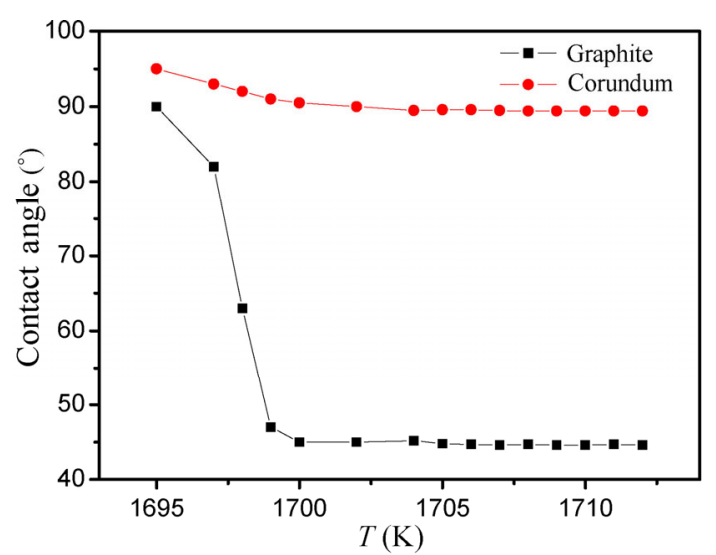

Fig. $10 \theta$ curves of molten silicon on polished graphite and corundum surface (Ar).

This equation illustrates the relationship between the static $\theta$, the gas-liquid surface tension $\left(\sigma_{\mathrm{LG}}\right)$, the solid-air interfacial tension $\left(\sigma_{\mathrm{SG}}\right)$, and the solid-liquid interfacial tension $\left(\sigma_{\mathrm{SL}}\right)$. With increasing $T, \sigma$ of the silicon liquid decreases linearly [20] and the liquid surface of the silicon liquid in the argon atmosphere decreases, thereby decreasing $\theta$. The penetration of the graphite interior by the silicon liquid passing through the graphite surface pores is another important factor that contributes to the $\theta$ reduction of the molten silicon on the graphite substrate [21]. This behavior yields greater reduction of the $\theta$ value on the graphite substrate than that on the corundum substrate. Further, at $1,700 \mathrm{~K}$, the molten silicon density changes abnormally. The coefficient of body expansion in this $T$ region is eight times $\left(8 \times 10^{-4}{ }^{\circ} \mathrm{C}^{-1}\right)$ that in the $T$ region beyond $1,700 \mathrm{~K}$, and is obviously caused by the change in $\theta$. Once $T$ reaches and exceeds $1,700 \mathrm{~K}, \theta$ remains almost constant.

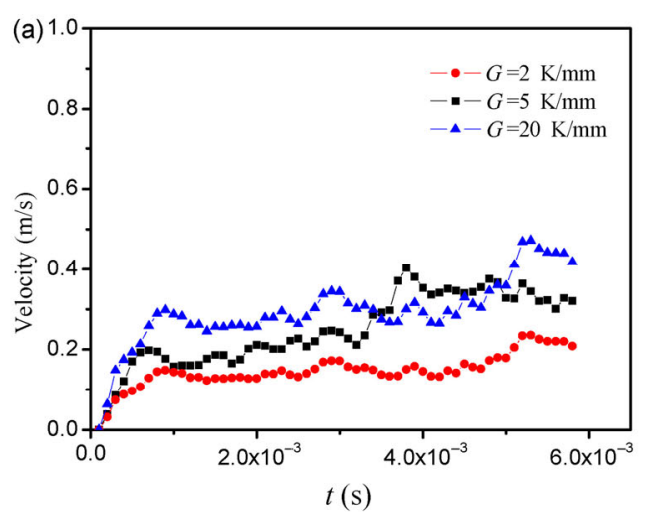

Figures 11(a) and 11(b) illustrate the variation trend of the average migration velocities of the molten silicon droplets on the corundum and graphite substrates, respectively, for $G=2,5$, and $20 \mathrm{~K} / \mathrm{mm}$. The initial $T$ is $1,700 \mathrm{~K}$ and the static $\theta$ values of the molten silicon on the graphite and corundum substrates are $45^{\circ}$ and $90^{\circ}$, respectively. With increasing substrate $G$, the average migration velocity increases and the instantaneous velocity on the graphite substrate $(t=0.009 \mathrm{~s})$ is as high as $1.8 \mathrm{~m} / \mathrm{s}$. Compared with the silicone-oil droplets, the differences in the thermal diffusivity, $\mu$, and $v$ values of the molten silicon droplets are greater.

Figures 12 and 13 illustrate the changes in the internal $T$ and flow fields of the droplets, respectively, during molten silicone droplet migration on the graphite substrate $(G=20 \mathrm{~K} / \mathrm{mm})$. In these figures, the colored lines represent isotherms, and the $T$ difference between each adjacent pair of lines is $6 \mathrm{~K}$. The substrate has a linear $G$; therefore, as the substrate heat is transferred inside the silicon droplets, a $\sigma$ surface tension gradient is generated at the gas-liquid interface that causes the droplets to move from the region with lower $\sigma$ to the region with higher $\sigma$. Further, a shear stress is generated from the high- to low- $T$ regions on the silicon liquid surface, as indicated by Eq. (6). The $\mu$ effect causes the formation of reflux in the silicon liquid.

Figure 13 shows that the thermocapillary convection influences the silicon liquid and forms two vortices with opposite directions. As the vortices change, the clockwise vortex undergoes greater development, which pushes the droplets toward the low- $T$ side. The

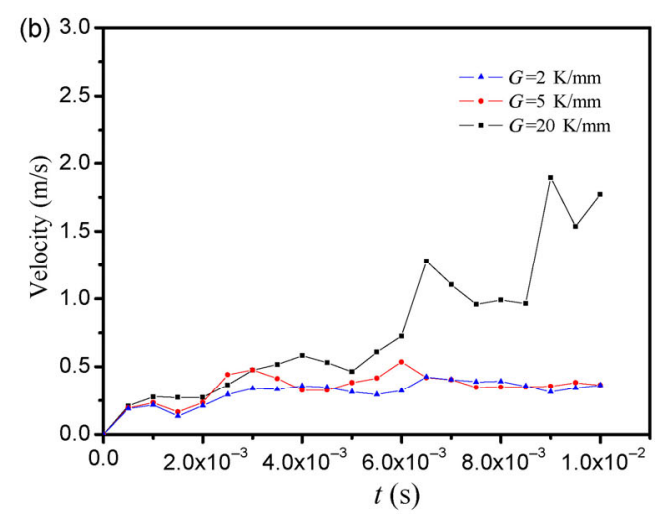

Fig. 11 (a) Thermocapillary migration velocities of molten silicon on (a) corundum $\left(\theta=90^{\circ}\right)$ and (b) graphite $\left(\theta=45^{\circ}\right)$ substrates under different $G$. 


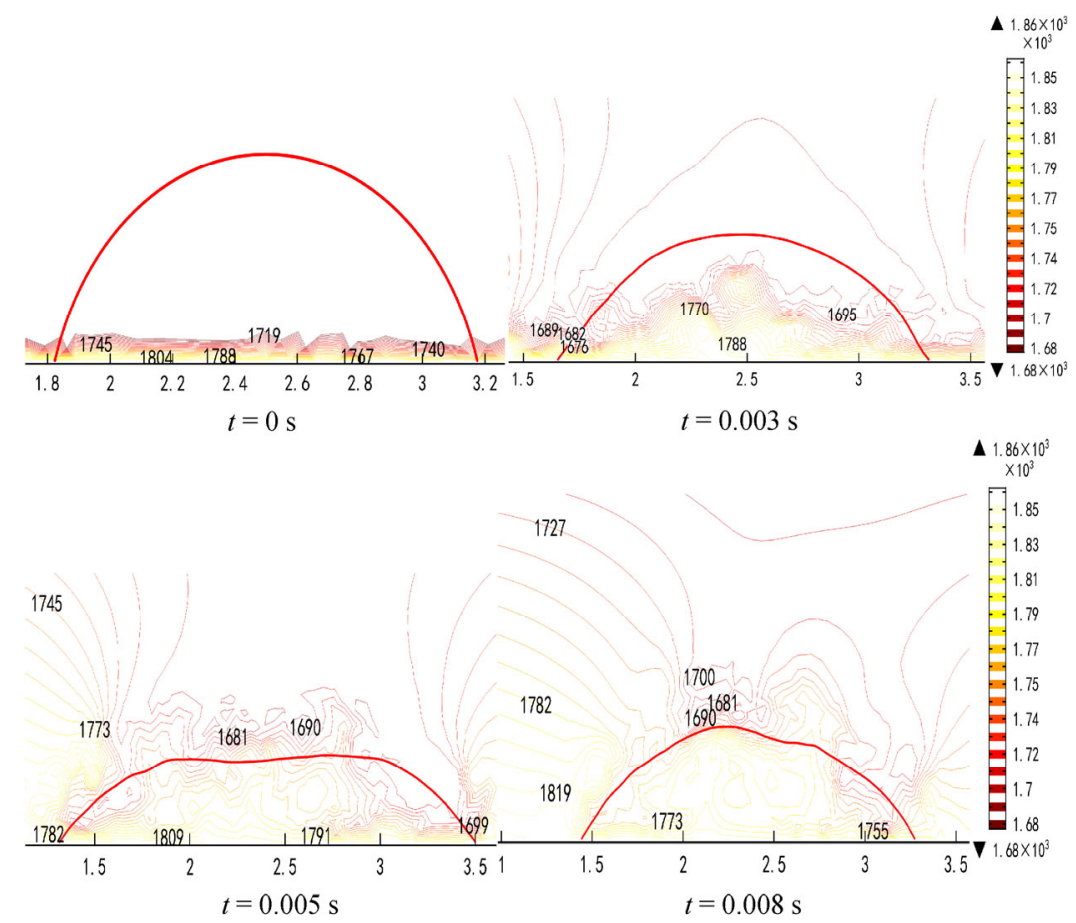

Fig. 12 Variation of $T$ field of molten silicon droplet on graphite substrate $\left(\theta=45^{\circ}\right)$ during motion process.

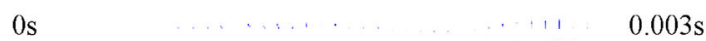
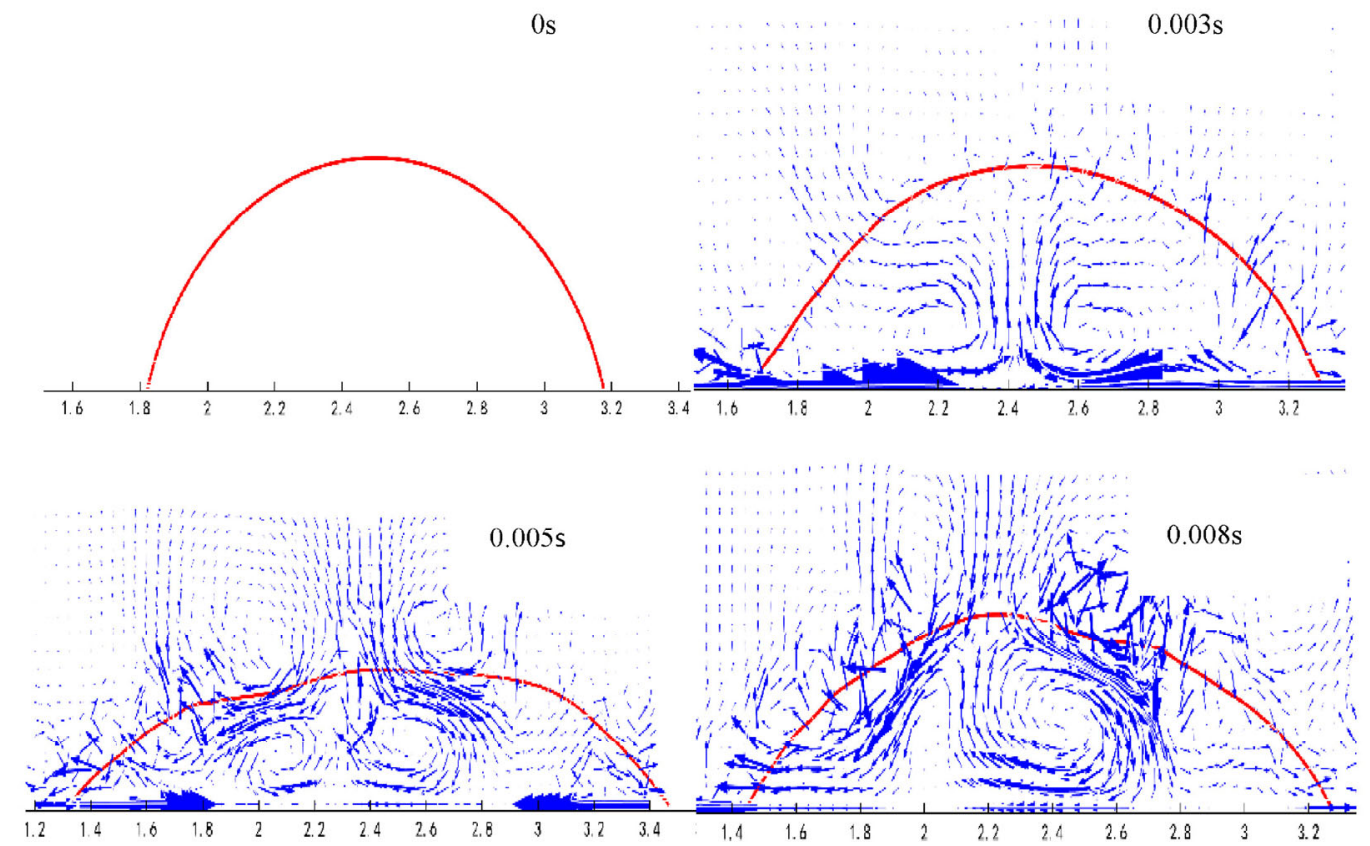

Fig. 13 Change of flow field of molten silicon droplet on graphite substrate $\left(\theta=45^{\circ}\right)$ during motion process.

development of the vortex affects the distribution of the internal $T$ of the silicon liquid. It can be seen from Fig. 12 that the distribution of the contours reflects the vortex shape, indicating coupling of the $T$ and flow fields. As the interfacial $T$ increases while the $\sigma$ decreases, the droplet has a tendency to spread.

Figures 14 and 15 show the change in the internal $T$ and the flow fields of the molten silicon droplets at $G=20 \mathrm{~K} / \mathrm{mm}$ on the corundum substrate. The $T$ difference between each pair of isotherm lines is $4 \mathrm{~K}$. 

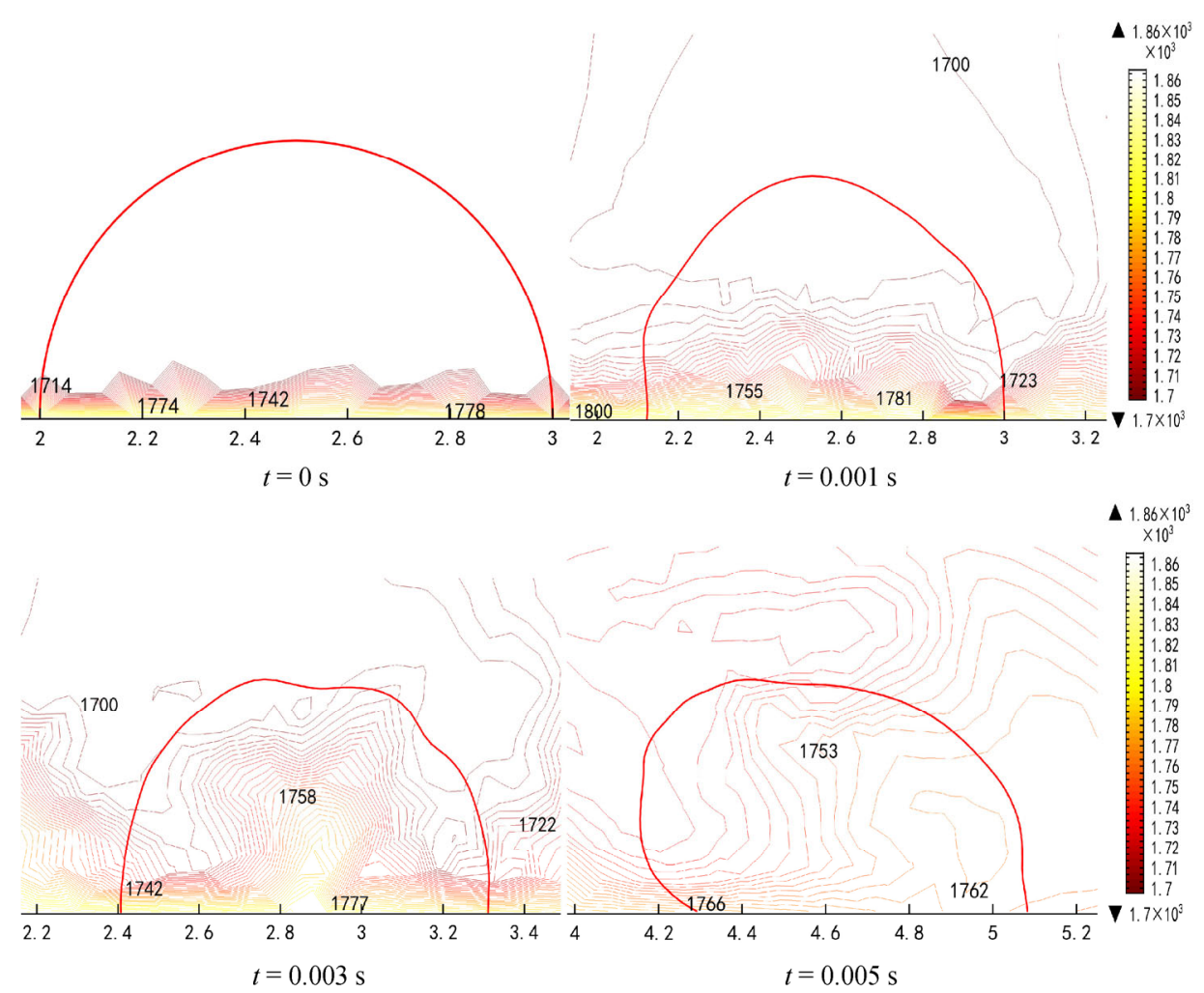

Fig. 14 Variation of $T$ field of molten silicon droplet on corundum $\left(\theta=90^{\circ}\right)$ substrate during motion process.

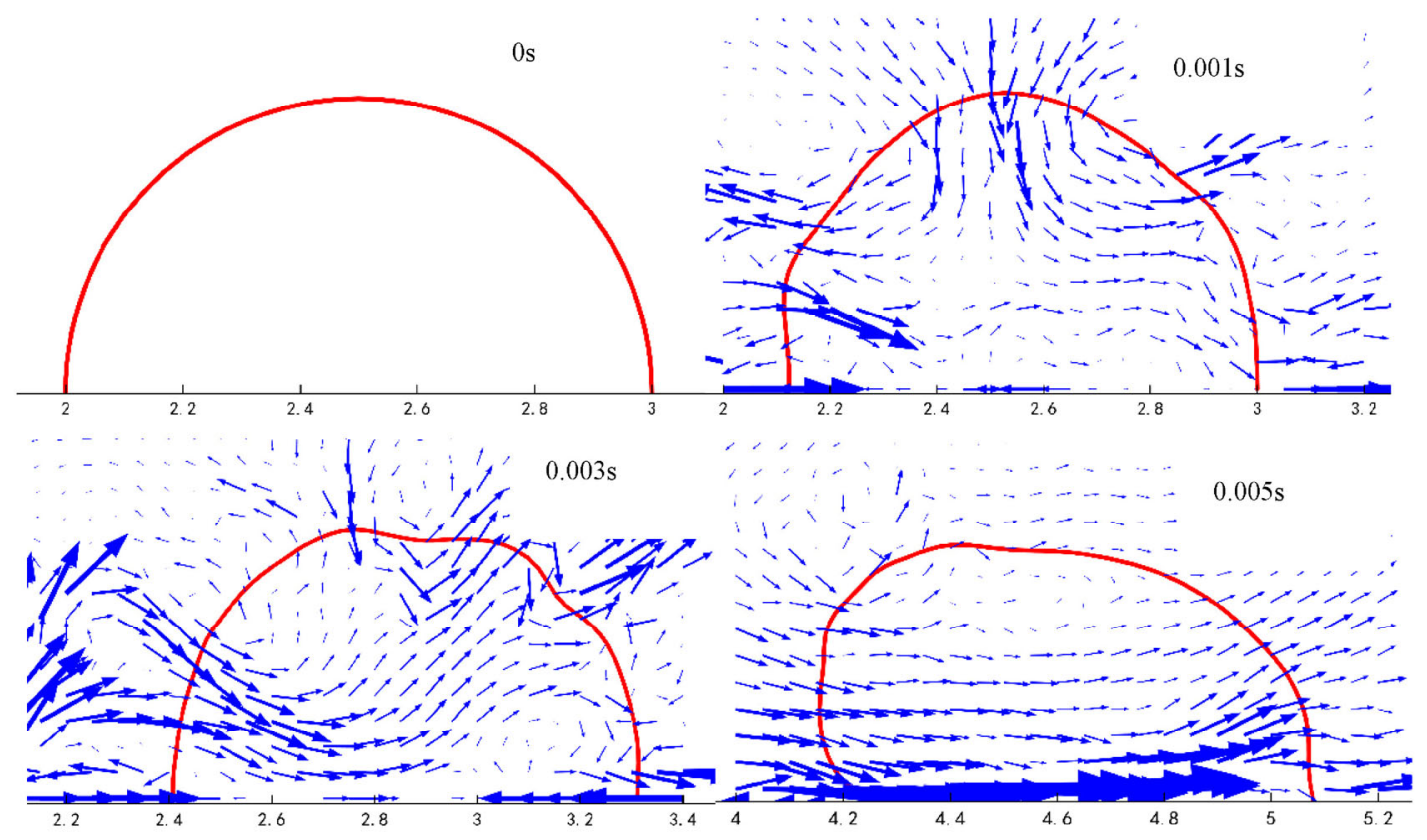

Fig. 15 Change of flow field of molten silicon droplet on corundum substrate $\left(\theta=90^{\circ}\right)$ during motion process.

Compared with the graphite substrate, the silicon liquid on the corundum substrate produces two vortices during the migration process. As the silicon liquid migrates to the cold side, the two vortices gradually disappear and form a stable laminar flow from the high- to low- $T$ regions. 


\section{Conclusion}

The thermocapillary actuation mechanism of a molten silicon droplet in a microchannel was studied via numerical computation, and the results were compared with experimental results. It was found that the variation in the surface tension from lower values on the hot side of the interface to higher values on the cool side generates a flow in the droplet, which exerts a hydrodynamic force on the solid surface in the direction of the applied temperature gradient. The solid surface, which is held immobile, exerts an equal and opposite reaction on the droplet that propels it in the opposite direction, i.e., toward cooler regions. Under suitable conditions, quasisteady motion can be achieved, wherein the net hydrodynamic force on the droplet is zero. Four horizontal vortex arrangements were generated during the migration process of the molten silicon droplet on a graphite substrate: two clockwise and two counterclockwise. During the same migration process, two vortices were formed on a corundum substrate. The vortices disappeared and gradually formed a stable laminar flow, and the droplet velocity became stable.

These results indicate that the actuation behavior of the droplet is strongly influenced by the temperature gradient. A higher temperature gradient generates higher droplet actuation velocity.

\section{Acknowledgements}

The authors appreciate the support from the Key Program of the National Natural Science Foundation of China (Grant No. 51335002) and Key support projects of strategic emerging industries in Jiangsu Province (Grant No. 2015-318).

Open Access: The articles published in this journal are distributed under the terms of the Creative Commons Attribution 4.0 International License (http:// creativecommons.org/licenses/by/4.0/), which permits unrestricted use, distribution, and reproduction in any medium, provided you give appropriate credit to the original author(s) and the source, provide a link to the Creative Commons license, and indicate if changes were made.

\section{References}

[1] Abdullah O I, Schlattmann J. Thermal behavior of friction clutch disc based on uniform pressure and uniform wear assumptions. Friction 4(3): 228-237(2016)

[2] Cheng G, Jiang S, Khosla T, Pesika N S, Ding J, Zhang Y, Wang Y. Synthesis of hard carbon/iron microspheres and their aqueous-based tribological performance under magnetic field. Tribol Lett 64: 48-56 (2016)

[3] Qian X, Xiang Y, Shang H, Cheng B, Zhan S, Li J. Thermaloxidation mechanism of dioctyl adipate base oil. Friction 4(1): 29-38 (2016)

[4] Heise R. Friction between a temperature dependent viscoelastic body and a rough surface. Friction 4(1): 50-64 (2016)

[5] Li H, Tian P, Lu H, Jia W, Du H, Zhang X, Li Q, Tian Y. State-of-the-art of extreme pressure lubrication realized with the high thermal diffusivity of liquid metal. ACS Appl Mater Interfaces 9 (6): 5638-5644 (2017)

[6] Brzoska J B, Brochardwyart F, Rondelez F. Motions of droplets on hydrophobic model surfaces induced by thermal gradients. Langmuir 9(8): 2220-2224 (1993)

[7] Brochard F. Motions of droplets on solid-surfaces induced by chemical or thermal gradients. Langmuir 5(2): 432-438 (1989)

[8] Tseng Y T, Tseng F G, Chen Y F, Chieng C C. Fundamental studies on micro-droplet movement by marangoni and capillary effects. Sensors \& Actuators A Physical 114(2): 292-301 (2004)

[9] Chen J Z, Troian S M, Darhuber A A, Wagner S. Effect of contact angle hysteresis on thermocapillary droplet actuation. Journal of Applied Physics 97(1): 257-1882 (2005)

[10] Li X, Guo F, Wang S, LIU C, WANG W. Behaviors of a micro oil droplet in an EHL contact. Friction 4(4): 359-368 (2016)

[11] Guo F, Wong P, Luo J. Guest editorial: Special issue on thin film lubrication. Friction 4(4): 277-279 (2016)

[12] Rhim W K, Ohsaka K. Thermophysical properties measurement of molten silicon by high-temperature electrostatic levitator: density, volume expansion, specific heat capacity, emissivity, surface tension and viscosity. Journal of Crystal Growth 208(1): 313-321 (2000)

[13] Olsson E, Kreiss G, Zahedi S. A conservative level set method for two phase flow II. Journal of Computational Physics 225(1): 785-807 (2005)

[14] Zahedi S, Kronbichler M, Kreiss G. Spurious currents in a finite- element based level set method for two phase flow. International Journal for Numerical Methods in Fluids 69(9): 1433-1456 (2009) 
[15] $\mathrm{Wu} \mathrm{Z} \mathrm{B.} \mathrm{Thermocapillary} \mathrm{migration} \mathrm{of} \mathrm{a} \mathrm{droplet} \mathrm{with} \mathrm{a}$ thermal source at large Reynolds and Marangoni numbers. International Journal of Heat \& Mass Transfer 75(16): 704-709 (2014)

[16] Le T L, Chen J C, Shen B C, Hwu F S, Nguyen H B. Numerical investigation of the thermocapillary actuation behavior of a droplet in a microchannel. International Journal of Heat \& Mass Transfer 83(3): 721-730 (2015)

[17] Felix Otto. Lubrication approximation with prescribed nonzero contact angle. Communications in Partial Differential Equations 23(11-12): 63-103 (1998)

[18] Hong S J, Chou T H, Liu Y Y, Sheng Y J, Tsao H K.

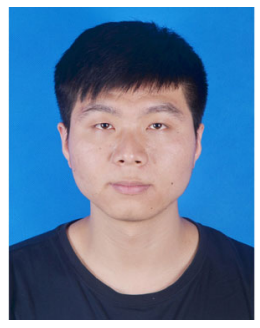

Tao SUN. He received his bachelor degree in mechanical engineering in 2014 from Changzhou University, Changzhou, China. After then, he was a master of Collaborative Innovation Center of Photovoltaic

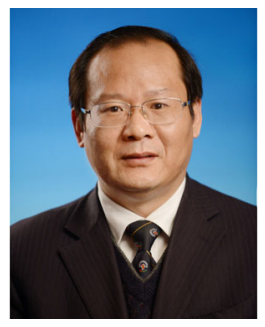

Jianning DING. He received his Ph.D. degree in mechanical engineering from Tsinghua University, China, in 2001. He joined Jiangsu University between 1991-2007. $\mathrm{He}$ is a research fellow in City University of Hong Kong from 2002 to 2003. His current position is a professor and vice president of Changzhou University. He is the director of Center for low-dimensional materials, micro-nano devices and system, director of Jiangsu
Advancing and receding wetting behavior of a droplet on a narrow rectangular plane. Colloid and Polymer Science 291(2): 347-353 (2013)

[19] Robert J. Contact angle, wetting, and adhesion: a critical review. Journal of Adhesion Science and Technology 6(12): 1269-1302 (1992)

[20] Shishkin A V, Basin A S. Surface tension of liquid silicon. Theoretical Foundations of Chemical Engineering 38(6): 660-668 (2004)

[21] Li J G, Hausner H. Wetting and infiltration of graphite materials by molten silicon. Scripta Metallurgica Et Materialia 32(3): 377-382 (1995)

Science and Engineering at the same university. He will obtain his master degree of engineering in mechanical engineering at Changzhou University in June, 2017. His research interests include manufacture and equipment of new energy materials and devices.

Collaborative innovation center of "Photovoltaic science and engineering" in 2011, director of Jiangsu Key Laboratory for Solar Cell Materials and Technology, the head of Science and Technology Innovation Team in Jiangsu Universities, and the chief scientist in the first level of young and middle-aged in "333 project" of Jiangsu province. His research areas cover the research of new energy materials, low-dimensional materials, micro-nano devices and system, and tribology. Above 500 academic papers and 6 books were published. 